\title{
Replacement behavior following deprivation of a saccharin solution or water'
}

JANIS W. DRISCOLL AND ROBERT B. LOCKARD UNIVERSITY OF WASHINGTON

The replacement properties of a need system (water) and an incentive system (saccharin) were compared following a 6-day period of fluid deprivation. While water deprivation was followed by complete replacement of the water deficit, deprivation of a saccharin solution was followed by substantial over-replacement of the water deficit. This result is interpreted as indicating an increase in the incentive value of the saccharin solution attributable to deprivation.

Much of the repetitive behavior of an animal is thought to be of two general types: Homeostatic or need systems which serve to maintain the internal condition of the animal in a relatively stable state; and incentive systems which are maintained by particular environmental stimuli. One major difference between these hypothetical response systems is the manner in which they ought to respond to deprivation. In a need system, where a reserve is being exhausted, there should be a compensatory increase in rate following deprivation which would continue until the deficit is replaced (Lockard, 1964). Incentive systems, on the other hand, would not be expected to show such an increase since no deficit has been created.

Pure examples of these types, however, may not exist. Water intake, although a relatively pure example of a need system with deficits accurately replaced through water ingestion (Adolph, 1964), certainly involves stimulus components, many of which may have incentive properties. Saccharin solutions are frequently considered examples of a need-irrelevant substance which is not only preferred to water (e.g., BeebeCenter et al, 1948) but has reinforcing properties as well (e.g., Sheffield \& Roby, 1950). But the taste of saccharin cannot be presented without the use of some solvent, and intake of saccharin is, therefore, not independent of water balance.

Although need and incentive properties of stimuli do not occur in isolation, it is possible to explore their interaction by examining the effects of the addition of a strong incentive component (saccharin) on replacement behavior following fluid deprivation. When a saccharin solution is the only available fluid, intake can be divided into need and incentive components by subtraction. An amount equal to the intake of watermaintained Ss under comparable conditions can be attributed to water need, while intake in excess of this amount by saccharin $S s$ is attributable to the extra incentive properties of the solution. If all fluid is removec, water Ss are deprived of only a need-related substance, while saccharin Ss are deprived not only of this substance, but also of the stimulation responsible for the extra intake. If the extra intake depends solely upon the physical properties of the incentive stimuli, animals re-provided with a saccharin solution after fluid deprivation should replace the water deficit, then resume the pre-deprivation intake rate, leaving the extra or incentive portion of the deficit unreplaced. Thus the purpose of this experiment was to determine to what extent the incentive value of a non-need stimulus may be affected by deprivaton.

\section{Method}

Twenty-four male Holtzman rats, 100 days of age, were maintained for 20 days on either water or a $.125 \%$ saccharin solution ( $\mathrm{N}=12$ in each condition). Six of the animals in each condition were then deprived of fluid for six days, after which the original fluid was returned. Control animals remained on the same fluid throughout the experiment.

Animals were housed in standard individual cages with ad lib access to food in food tubes and fluid in Wahmann No. LC-237B reservoir bottles. These bottles were cleaned every four days. The temperature and humidity of the experimental room were maintained at $78^{\circ} \mathrm{F}$ and $50 \% \mathrm{RH}$. A light-dark cycle of $12 \mathrm{hr}$. on$12 \mathrm{hr}$. off was in effect. Measurements were taken during the early part of the light phase of the cycle. Extraneous noise was masked by the continuous running of humidifiers.

The saccharin solution contained $.125 \mathrm{gm}$ of sodium saccharin in $100 \mathrm{ml}$ of solution. Tap water was used in the saccharin solution which was prepared $8000 \mathrm{ml}$ at a time and stored in a large glass container. New solution was mixed every four days. Solution to be used the following day was transferred to one-liter plastic bottles $24 \mathrm{hr}$. before use. Tap water was stored in similar plastic bottles $24 \mathrm{hr}$. before it was used. Resulfs

The results of the replacement period are summarized in Fig. 1. Data are presented in the form of deficits obtained as follows: Each point represents the difference between the mean intake of the deprived group and the mean intake of the appropriate control group for that day. These differences, accumulated for the deprivation and replacement periods, show the extent of the deficit for any day during deprivation and replacement. Complete replacement would be indicated by a deficit of zero at the end of replacement. Because the replacement concept requires the use of cumulative data, conventional statistical techniques do not apply in an unambiguous fashion. Therefore, in 


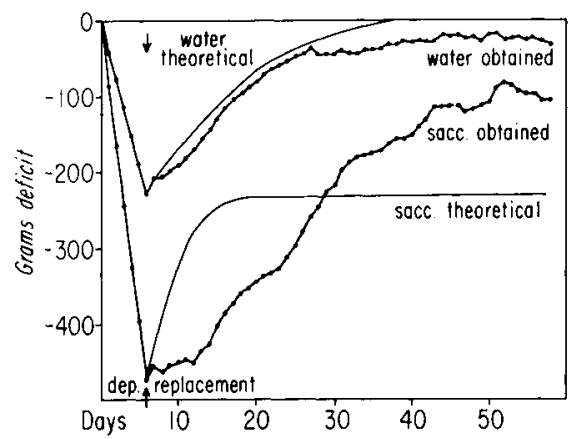

Fig. 1. Replacement of water or a saccharin solution following fluid deprivation.

order to correct systematic differences between control and experimental groups to make replacement data comparable, proportional constants were calculated, based on intake during the baseline period, and applied to the replacement data. These constants were 1.01 and .99 for saccharin and water-replacing groups respectively.

As can be seen in Fig. 1, when water was the replacement fluid, the deficit incurred during deprivation was almost completely replaced. Animals replacing with saccharin, however, over-replaced the estimated water portion of the fluid deficit by approximately $150 \mathrm{gm}$ before resuming the pre-deprivation rate. This over-replacement was due to continued high intake of saccharin for approximately 15 days after water-replacing animals had resumed the pre-deprivation rate. These inflated intakes continued after food intake had returned to the pre-deprivation rate and are, therefore, not entirely attributable to food deprivation, although it may have been involved. Both food intake and body weight were measured daily but are not reported. These data were consistent with the conclusions drawn from the fluid data.

\section{Discussion}

Fig. 1 also presents theoretical water and saccharin replacement functions. These functions are based on the following assumptions: (1) That the intake of undeprived water animals provides an estimate of daily water need $\left(W_{N}\right)$ and water deficit $(D)$, where $D=\Sigma W_{N}$ for the deprivation period; and (2) that all fluid consumed in excess of daily water need is utilized for replacement of the water deficit. Thus, $A_{R}=I-W_{N}$, where $A_{R}=$ the amount replaced and $I=$ the intake on any day during replacement.

The water function assumed, in addition, that .1 of the remaining water deficit $\left(D_{R}\right)$ is replaced each day, where $D_{R}=D-\Sigma A_{R}$ and $I=W_{N}+.1 D_{R}$. As can be seen, this corresponded approximately to the obtained data, and a different constant would have provided a closer fit.
The theoretical saccharin function, in accordance with assumption 2 above, was calculated as follows: $A_{R}=I_{S}-W_{N}$, where $I_{S}=$ the saccharin intake of deprived animals. It was also assumed that when the water deficit had been replaced $\left(\Sigma A_{R}=D\right)$, the saccharin intake would resume the pre-deprivation rate.

It should be noted that a direct comparison between theoretical and obtained saccharin functions cannot be made since the theoretical function is based on differences between water intake and saccharin intake, while the obtained saccharin function is based upon differences in the saccharin intake of deprived and undeprived animals. The theoretical saccharin function is presented to provide an estimate of the point at which the saccharin-replacing animals had consumed sufficient fluid in excess of daily water need to have replaced the water deficit and would be expected to resume the pre-deprivation rate.

It is obvious from Fig. 1 that saccharin consumption did not resume the pre-deprivation rate when the estimated water deficit had been replaced. This lack of correspondence between theoretical expectations and obtained data leads to the following conclusions. It is clear that the incentive value of the saccharin solution, defined in terms of intake in excess of water need, is enhanced by deprivation. This result provides support for Bolles' (1958) view that the incentive value of a stimulus may be a function of the period of deprivation and brings into question the classical distinction between need systems and incentive systems. However, since little information is available concerning the effect of length of deprivation on subsequent intake of an incentive, further experimentation involving variation in both the length of the deprivation period and the incentive value of the replacement fluid will be required to clarify these issues.

\section{References}

Adolph, E. F. Regulation of body thirst content through water ingestion. In Matthew J. Wayner (Ed.), Thirst. New York: The Macmillan Co., 1964. Pp. 5-14.

Beebe-Center. J. G., Black, Percy, Hoffman, A. C., \& Wade, Marjorie. Relative per diem consumption as a measure of preference in the rat. $J$. comp. physiol. Psychol., 1948, 41, 239-251.

Bolles, Robert C. Comments on Professor Spence's paper. In Marshall R. Jones (Ed.), Nebraska symposium on motivation. LincoIn: University of Nebraska Press, 1958. Pp. 108-110.

Lockard, Robert B. A method of analysis and classification of repetitive response systems. Psychol. Rev., 1964, 71, 141-147.

Sheffield, Fred D., \& Roby, Thomton, B. Reward value of a nonnutritive sweet taste. J. comp. physiol. Psychol., 1950, 43, $471-481$.

\section{Note}

1. This research was supported by Grant GB-3141 from the National Science Foundation and NIMH predoctoral fellowship No. 5-F 1-MH$21,960-03$ (PS). 